Zellen (EPC), senkt die mitochondriale Produktion reaktiver Sauerstoffspezies und verstärkt die Neoangiogenese im Myokard.

\section{Pilotstudie bestätigt positive} hämodynamische Effekte Prof. Veselin Mitrovic, Bad Nauheim, und Mitarbeiter konnten in einer Pilot- studie an fünf Patienten mit Herzinsuffizienz (NYHA II-IV) positive hämodynamische Effekt von PETN bestätigen. Sowohl akut nach einer Einzelgabe von 80 mg PETN als auch chronisch nach siebentägiger Therapie mit $2 \times 80 \mathrm{mg}$ täglich beobachteten sie eine Abnahme des Pulmonalarteriendrucks und des pulmonalen Kapillardrucks. Auch unter Belastung wurde eine Drucksenkung im kleinen Kreislauf beobachtet. Dass der Effekt auch nach sieben Tagen nicht abgeschwächt war, bestätigt die fehlende Toleranz unter PETN.

- Dr. med. Angelika Bischoff

Quelle: 17. PETN Expertentreffen, München, 16. Oktober 2010 (Veranstalter: Actavis)

\title{
Symptom Sodbrennen
}

\section{Funktionelle Dyspepsie oder Refluxösophagitis?}

- Beschwerden im Oberbauch wie Sodbrennen, frühes Sättigungsgefühl, Völlegefühl und Aufstoßen können sowohl organischer wie auch funktioneller Art sein. In der Regel sind die Symptome aber nicht eindeutig einer Krankheit zuzuordnen. Lautet das Hauptsymptom "Sodbrennen", werden meist reflexartig Protonenpumpenhemmer (PPI) verordnet, da ein Säureüberschuss vermutet wird.

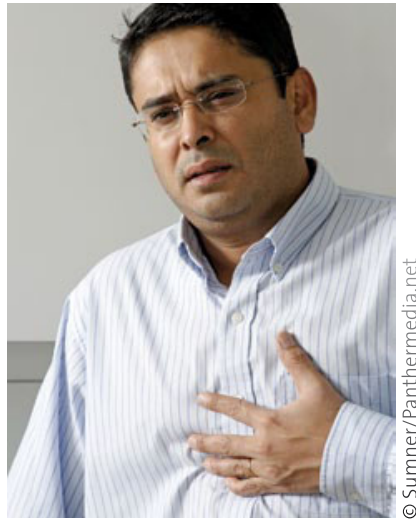

Thoraxschmerz und Aufstoßen: das spricht für eine Refluxkrankheit.
Allerdings klagen auch Patienten mit funktioneller Dyspepsie über Sodbren- nen. PPI helfen hier aber nicht, da die Ursache nicht „zu viel Säure“ ist, sondern „Säure am falschen Ort", zum Beispiel durch gestörte Magenmotilität. Schätzungen zufolge werden bis zu $50 \%$ der PPI nicht indikationsgerecht eingesetzt.

\section{Checkliste für die Praxis}

Die therapeutischen Konsequenzen der Differenzierung zwischen funktioneller Dyspepsie und Refluxösophagitis sind erheb- lich. Während bei Letzterer der Einsatz eines Säureblockers in der Regel notwendig ist, kann die Dyspepsie meist mit einem Phytopharmakon wie Iberogast ${ }^{\circledR}$ erfolgreich behandelt werden.

Um bei der Erstbefragung des Patienten sicher zwischen funktioneller Dyspepsie und Refluxösophagitis unterscheiden zu können, hat das Kompetenzteam Magen unter Federführung von Prof. Hans-Dieter Allescher, GarmischPartenkirchen, eine strukturierte Diagnose-Checkliste erarbeitet - mit charakteristischen Symptombildern und zielführenden Fragestellungen für den Praxisalltag.

- Steigerwald

\section{Tabelle 1}

\section{Klärende Fragen an den Patienten}

\begin{tabular}{|c|c|c|}
\hline & $\begin{array}{l}\text { Funktionelle } \\
\text { Dyspepsie }\end{array}$ & $\begin{array}{l}\text { Reflux- } \\
\text { krankheit }\end{array}$ \\
\hline $\begin{array}{l}\text { (1) Treten die Beschwerden vor allem nachts auf? } \\
\text { Wachen Sie deshalb auf? }\end{array}$ & Nein & $\mathrm{Ja}$ \\
\hline $\begin{array}{l}\text { (2) Treten die Beschwerden vor allem im } \\
\text { Zusammenhang mit Mahlzeiten auf? }\end{array}$ & $\mathrm{Ja}$ & Nein \\
\hline $\begin{array}{l}\text { (3) Leiden Sie derzeit unter einer zu } \\
\text { großen Arbeitsbelastung? }\end{array}$ & Nein & $\mathrm{Ja}$ \\
\hline (4) Fühlen Sie sich seelisch gestresst? & $\mathrm{Ja}$ & Nein \\
\hline $\begin{array}{l}\text { 5 Kommt es bei Ihnen häufig zu } \\
\text { Thoraxschmerz und Aufstoßen? }\end{array}$ & Nein & $\mathrm{Ja}$ \\
\hline $\begin{array}{l}\text { (6) Erleben Sie es häufig, dass Sie } \\
\text { sich schon nach dem zweiten Bissen } \\
\text { satt und gebläht fühlen? }\end{array}$ & $\mathrm{Ja}$ & Nein \\
\hline $\begin{array}{l}\text { (7) Brauchen Sie eine spezielle Diät, } \\
\text { um Beschwerden zu vermeiden? }\end{array}$ & $\mathrm{Ja}$ & Nein \\
\hline $\begin{array}{l}8 \text { Meiden Sie speziell Bohnenkaffee } \\
\text { und Speisen mit „schlechtem“ oder kaltem Fett? }\end{array}$ & Nein & $\mathrm{Ja}$ \\
\hline
\end{tabular}

Magen up to date

Kompetenzteam Magen gibt praxisnahe Hilfestellungen

Mit dem Periodikum „Magen up to date "lieferte das Kompetenzteam Magen einen praxisnahen Leitfaden für die fundierte Diagnose und Therapie von Oberbauchbeschwerden. Das Periodikum kann bei Steigerwald Arzneimittelwerk $\mathrm{GmbH}$, Havelstr. 5 , 64295 Darmstadt, telefonisch unter 06151 - 3305178 oder per E-Mail an most@steigerwald.de kostenlos angefordert werden.

Mitglieder des Kompetenzteams Magen sind Prof. Hans-Dieter Allescher, Garmisch-Partenkirchen, Prof. Peter Malfertheiner, Magdeburg, Dr. Martin Strauch, München, und Dr. Ulrich Koczian, Augsburg. 